\title{
Oxidative environment and redox homeostasis in plants: dissecting out significant contribution of major cellular organelles
}

\author{
Priyanka Das ${ }^{1}$, Kamlesh K. Nutan ${ }^{1}$, Sneh L. Singla-Pareek ${ }^{2}$ and Ashwani Pareek ${ }^{\text {* }}$ \\ 1 Stress Physiology and Molecular Biology Laboratory, School of Life Sciences, Jawaharlal Nehru University, New Delhi, India \\ 2 Plant Molecular Biology, International Centre for Genetic Engineering and Biotechnology, New Delhi, India
}

Edited by:

Naser A. Anjum, University of

Aveiro, Portugal

Reviewed by:

Naser A. Anjum, University of

Aveiro, Portugal

Brahma B. Panda, Berhampur

University, India

Yehia ElTemsah, Cairo University,

Egypt

\section{*Correspondence:}

Ashwani Pareek, Stress Physiology

and Molecular Biology Laboratory,

School of Life Sciences, Jawaharlal

Nehru University, Room No. 401,

New Delhi 110067, India

e-mail:ashwanip@mail.jnu.ac.in
Plant cells are often exposed to oxidative cellular environments which result in the generation of toxic reactive oxygen species (ROS). In order to detoxify the harmful ROS, plants have evolved various strategies including their scavenging and antioxidant machinery. Plant cells contain many enzymatic and non-enzymatic antioxidants which aid in removing the toxic oxygen molecules. Various antioxidant molecules localized within different cellular compartments play crucial role(s) during this process, which includes both redox-signaling and redox-homeostasis. The present review gives an overview of cellular oxidative environment, redox signaling operative within a cell and contributions of major cellular organelles toward maintaining the redox homeostasis. Additionally, the importance of various antioxidant enzymes working in an orchestrated and coordinated manner within a cell, to protect it from stress injury has been presented. We also present the state-of-the-art where transgenic approach has been used to improve stress tolerance in model and crop species by engineering one or more than one of these components of the ROS scavenging machinery.

Keywords: redox homeostasis, reactive oxygen species, ascorbate peroxidase, catalase, superoxide dismutase, abiotic stress

\section{INTRODUCTION}

Plants are obligate aerobic organisms like animals and they require oxygen for mitochondrial energy production. Furthermore, plants can deal with much higher concentration of oxygen as the green tissues of plants continuously produce oxygen through the process of photosynthesis during day time. In plants, the green leaves contain 2.5 fold higher oxygen concentration than the non-green parts like root. In both green and non-green parts, the oxygen concentration is much higher than the oxygen concentration found in animal cells (Vanderkooi et al., 1991). Plant tissues experience wide oxygen fluctuations under abiotic stress conditions, making the surroundings strongly hypoxic (Bailey-Serres and Voesenek, 2008). Plant seeds also experience huge oxygen variations. When green young seeds are photosynthetically dynamic, the light-dark reaction generates large and quick variations in the internal oxygen concentrations of Brassica napus. The variation ranges from strong hyperoxia $(>700 \mu \mathrm{M}$ in day) to severe hypoxia ( $<1 \mathrm{mM}$ in night). Similar situations have also been observed in many other species (Borisjuk and Rolletschesk, 2008).

As a natural result of the oxygen metabolism, plants continuously produce reactive oxygen molecules/species (ROS) like superoxides and peroxides (Panda et al., 2013; Kangasjarvi and Kangasjarvi, 2014; Vainonen and Kangasjarvi, 2014). Although, high concentration of these ROS has negative effect on plants, specific concentrations of ROS play vital roles in cell signaling. Continual exposure to ROS creates an oxidative environment which affects the redox balance of the cell. Alterations in redox state in intracellular region also have a major consequence on cell functions as various cellular signaling pathways regulating cell division and stress reaction systems are sensitive to redox situation (Chiu and Dawes, 2012). Severe redox situation often leads to senescence and death of the cell and ultimately the organism.

Antioxidants with low molecular weight like ascorbate, tocopherol and glutathione, are redox buffers which act as enzyme cofactors and play crucial roles in defense, cell proliferation to aging and death (Tokunaga et al., 2005). Antioxidants supply necessary information on redox state of the cell, and they control the expression of the genes linked with abiotic and biotic stresses to increase stress defense. Maintaining the level of these ROS at a balanced state is always crucial for plants and for this purpose, plants have adopted various cellular mechanisms. Growing facts suggest models for redox homeostasis where the antioxidant-ROS communications play as a metabolic interface for signals derived from metabolism and from the environment. Present topic talks about the roles of various cellular organelles in maintaining the redox homeostasis in plant cells and ultimately helping toward abiotic stress tolerance in plant.

\section{OXIDATIVE ENVIRONMENT, ANTIOXIDANT INTERACTIONS AND REDOX SIGNALING IN PLANT CELL}

Oxidative environment is generated when ROS is produced by a specific or by combination of multiple stresses (Thorpe et al., 2004). Process of generation of oxygen in cell has been mentioned 
or reviewed by many researchers (Aung-Htut et al., 2012; Kumar et al., 2012; Khanna-Chopra et al., 2013; Ghosh et al., 2014). The first product of specialized water producing reactions catalyzed by oxidases is superoxide and from superoxide, other ROS are produced subsequently. Singlet oxygen is also produced while capturing of light and process of photochemistry is going on. Numerous enzymatic processes generate superoxide $\left(\mathrm{O}_{2}^{-}\right)$ or hyderogen peroxide $\left(\mathrm{H}_{2} \mathrm{O}_{2}\right)$. Most of the cellular compartments (chloroplast, mitochondria, peroxisome, and cytoplasm) in higher plants participate in the generation of ROS inside the cell (Figure 1).

Abiotic stresses like drought, salinity, low temperature or high temperature often limit the $\mathrm{CO}_{2}$ fixation and reduce the generation of $\mathrm{NADP}^{+}$through Calvin cycle. Therefore, over-reduction of the photosynthetic electron transport chain (ETC) is occurred which generates superoxide radicals and singlet oxygen in the chloroplasts ( $\mathrm{Li}$ and Jin, 2007). To avoid the over-reduction of the ETC under stress conditions, higher plants modified the pathway of photorespiration to regenerate $\mathrm{NADP}^{+}$(Shao et al., 2006). $\mathrm{H}_{2} \mathrm{O}_{2}$ is generated in the peroxisomes as a by-product of photorespiratory pathway (Foyer and Noctor, 2005).

To control the production of the highly toxic ROS, higher plants possess enzymatic and non-enzymatic antioxidant defense systems that help in scavenging of ROS and protection of plant cells from oxidative damage (Foyer and Noctor, 2005). High accumulation of non-enzymatic ROS scavengers, and different biochemical properties, different localization and differential inducibility at the transcript or protein level of antioxidant enzymes provide the antioxidant systems, a very flexible unit that can control ROS accumulation temporally and spatially (Foyer and Noctor, 2005; Shao et al., 2006). The antioxidant enzymes such as catalase (CAT), superoxide dismutase (SOD), glutathione reductase (GR), ascorbate peroxidase (APX), GR and monodehydroascorbate reductase (MDAR) play major role in scavenging the toxic ROS inside the plant cell along with the non-enzymatic ROS scavengers like ascorbic acids and reduced glutathione (Figure 1).

Plants have evolved inbuilt mechanism to sense, transduce, and translate ROS signals into appropriate cellular responses. This particular process requires the existence of redox-sensitive proteins that can take part both in oxidation and reduction reactions and may regulate the switching-on or -off depending upon the cellular redox state (Shao et al., 2006). The redoxsensitive proteins are directly or indirectly oxidized by ROS via the ubiquitous redox-sensitive molecules, such as thioredoxins (Trxs) or glutathione (Nakashima and Yamaguchi-Shinozaki, 2006). The cellular metabolism under oxidative stress is directly modulated by redox-sensitive metabolic enzymes, but the redoxsensitive signaling proteins complete their action via downstream

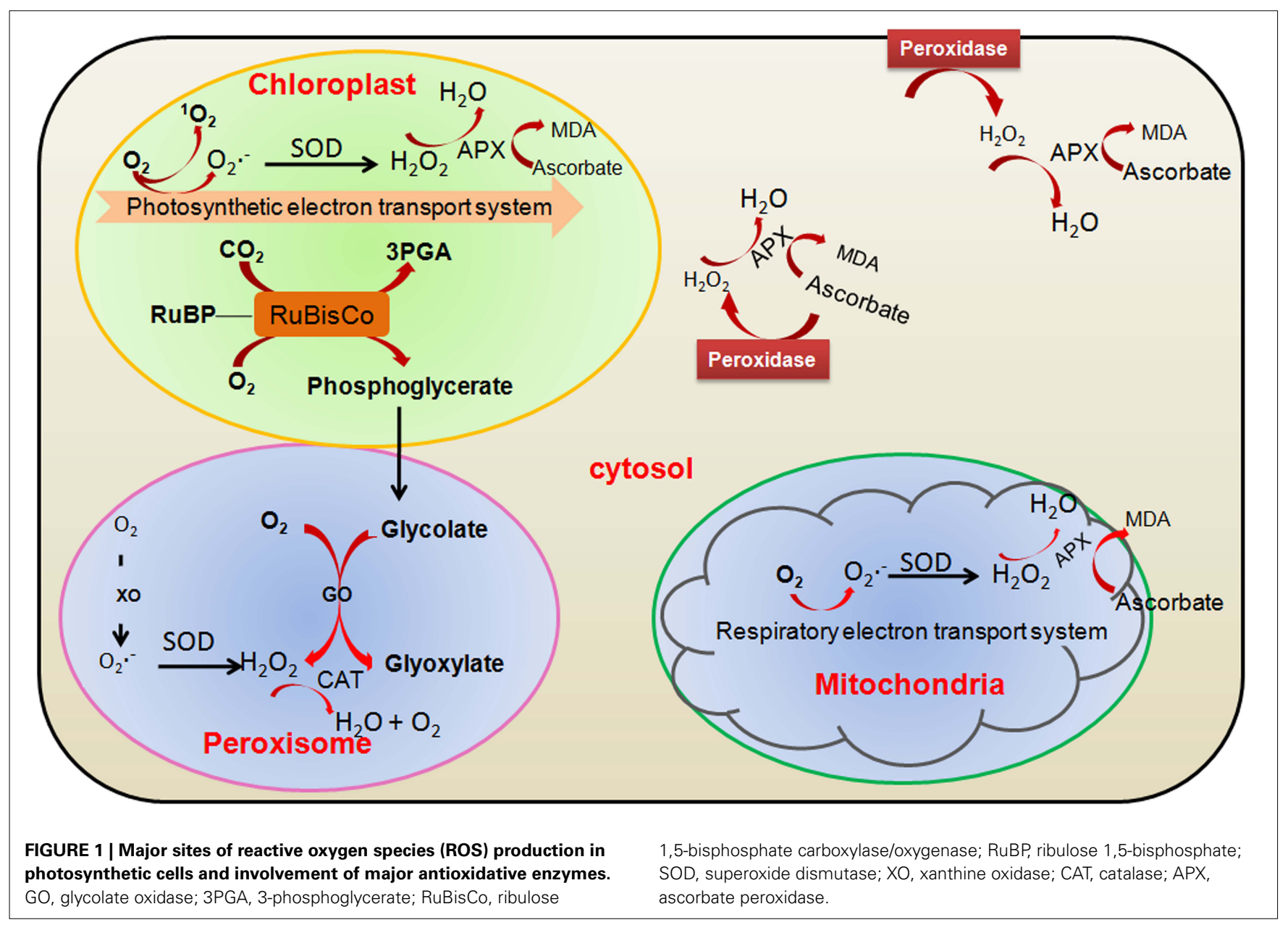


signaling components, such as phosphatases, kinases and transcription factors (Foyer and Noctor, 2005; Li and Jin, 2007). Molecular mechanisms of redox-sensitive regulation of protein have also been explained for plants and other living organisms (Cvetkovska et al., 2005; Foyer and Noctor, 2005). ROS mediated signaling involves hetero-trimeric G-proteins and MAP kinase regulated protein phosphorylation and protein Tyr phosphatases (Pfannschmidt et al., 2003; Foyer and Noctor, 2005; Kiffin et al., 2006). Mitogen-activated protein kinase (MAPK) cascades are mainly engaged by eukaryotes which have got much concentration for research since long years. The minimal signal transduction unit is considered to have a stimulus-activatable MAP kinase kinase kinase (MAPKKK), a MAP kinase kinase (MAPKK), a MAP kinase (MAPK) and their downstream targets. A chronological phosphorylation-activation process begins which transmit the signal from the MAPKKK to the target, which may be a transcription factor (TF) whose activity and localization is influenced by phosphorylation. The proportions of phosphorylation activation and transmission indicate that MAPKKKs can be activated by particular stimuli and the signaling pathways may congregate at the MAPKK level of the cascade. A single MAPKK could then phosphorylate several MAPKs. The signaling through MAPKKKs and MAPKKs could continue through other mechanisms as well besides phosphorylation of their direct downstream targets (Figure 2). This occurs with the Arabidopsis MAPKKK, MEKK1, which may phosphorylate the WRKY53 TF and additionally, bind to its promoter which functions as an activator for transcription (Miao et al., 2007). Salinity and cold reactive MEKK1-MKK1/2-MPK4/6 signaling cascade (Teige et al., 2004), which appears to have a bi-directional communication with ROS: the MEKK1 protein have been reported to be stimulated and stabilize by $\mathrm{H}_{2} \mathrm{O}_{2}$ and also the MAPK components -MPK4 and MPK6 have been found to be activated by ROS and various abiotic stresses (Teige et al., 2004).

In higher plants, the biochemical and structural basis of kinase pathway activation by ROS is yet to be established, but thiol oxidation probably has a key contribution here (Yabuta et al., 2004; Foyer and Noctor, 2005). Stromal ferredoxin-thioredoxin system is the well-known redox signal transduction system in plants which functions during photosynthetic metabolism of carbon. Signal transmission occupies disulfide-thiol alteration in target enzymes (Yabuta et al., 2004). Increasing authentication shows that plant hormones are situated downstream of the ROS signal. Induction in accumulation of stress hormone, such as salicylic acid and ethylene, is caused by $\mathrm{H}_{2} \mathrm{O}_{2}$ (Kiffin et al., 2006). Plant hormones are not only placed downstream to the ROS signal, ROS also play a role as secondary messengers in many hormone signaling pathways (Kwon et al., 2006). It indicates that backward or forward interactions may possibly occur between different hormones and ROS (Rio et al., 2006; Terman and Brunk, 2006).

\section{REDOX HOMEOSTASIS IN PLANTS}

Concurrent occurrence of both reduced and oxidized forms of electron transporters is required for competent flux through electron transport cascades in plants. This condition is known as redox poising and it involves an uninterrupted change of electrons to oxygen molecule from diverse sites in the respiratory and photosynthetic electron transport chains. The reactive character

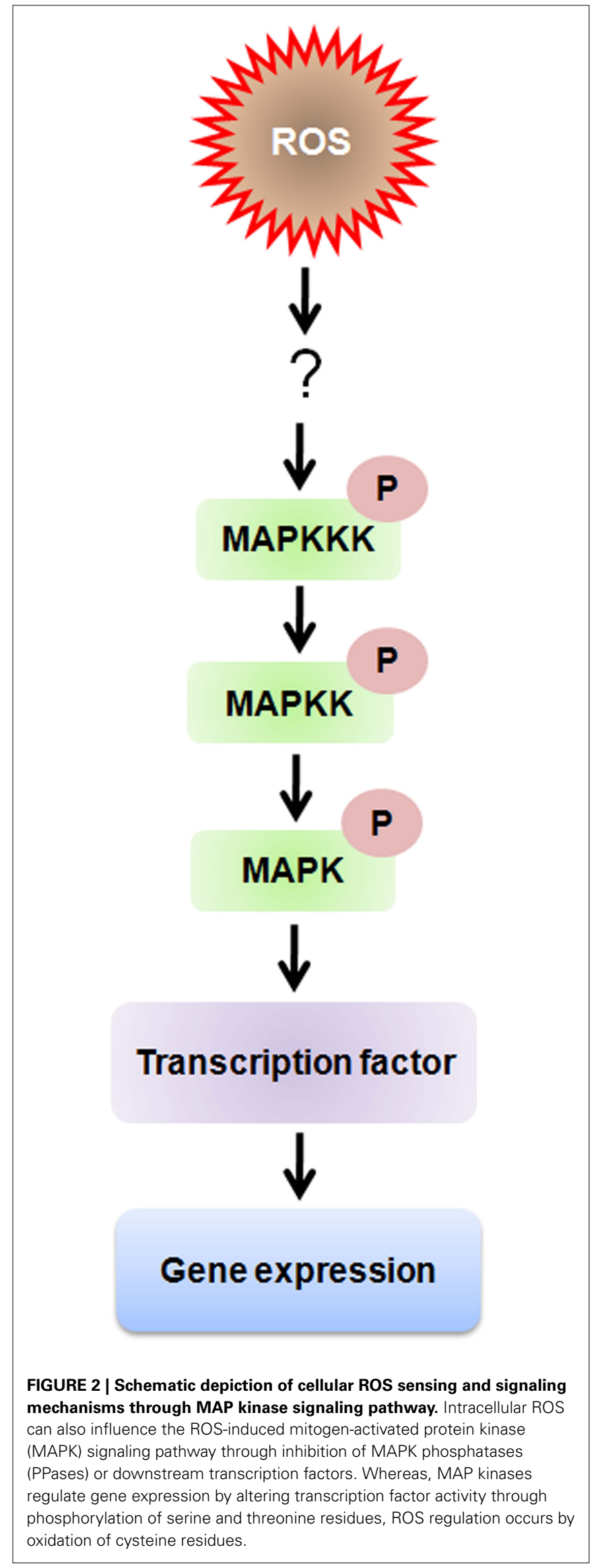


of these ROS means not only that their increasing concentration should be controlled but also that they are capable to play as signaling molecules. The level of accretion of ROS is determined by the antioxidative system which enables cells to preserve the cellular components in an active state for metabolism.

Similar to many other aerobic animals, plants preserve most cytoplasmic thiols in the reduced (2SH) condition, as the low thiol disulfide redox potential imposed by millimolar concentrations of glutathione is the thiol buffer. Nevertheless, plant cells produce high concentrations of ascorbate, an added hydrophilic redox buffer which gives strong defense against oxidative stress. Redox homeostasis is directed by the large pools of these antioxidants which maintain the level of reductants and oxidants in a balanced state. Tochopherols (Vitamin E) are important liposoluble redox buffers produced by the plants. Although, tochopherol is known as a major singlet oxygen scavenger, it also can efficiently scavenge other ROS (Foyer and Noctor, 2005). Furthermore, as the tocopherol redox couple has an additional constructive midpoint potential than the ascorbate pool, it further amplifies the range of efficient superoxide scavenging. The capacity of the glutathione, ascorbate and tocopherol pools, to play as redox buffers in plant cells, is one of their significant characteristics.

ROS signaling pathways are made achievable by homeostatic regulation accomplished by antioxidant redox buffering. As the antioxidants constantly process ROS, they decide the duration and the specificity of the signal of ROS. Plant cells usually handle the high rate of generation of ROS in a very careful way. Even though, cellular oxidation is significant in all biotic and abiotic stress reactions, the level and physiological consequence of oxidative injury is arguable. For instance, plants with low cytosolic APX and CAT activities show less severe stress indications than the plants which require either one of these enzymes (Rizhsky et al., 2002). It has also been established that cell death mediated by singlet oxygen is not a direct consequence of damage per se but somewhat is genetically programmed through the EXECUTOR1 pathway (Wagner et al., 2004). Moreover, plants adapt very well to depletion of antioxidants by signaled induction of other defense systems such as: tocopherol-deficient Arabidopsis vte mutant seedlings have high amounts of lipid peroxides, but the mature plants show slightly abnormal phenotype (Kanwischer et al., 2005). Furthermore, it has been well-established that most of the cellular organelles play important roles to maintain the redox homeostasis in the plant cell (Andreev, 2012; Ferrández et al., 2012; Lázaro et al., 2013). Following section describes the contribution of major cell organelles toward maintaining cell redox homeostasis under oxidative environment.

\section{INVOLVEMENT OF MAJOR CELLULAR ORGANELLES IN MAINTAINING REDOX HOMEOSTASIS IN PLANT CELL CONTRIBUTION OF CHLOROPLAST}

Dithiol-disulphide exchange based post translational alteration comprises a fast and reversible mechanism of regulation in a cell. Thus, it allows the competent adaptation of metabolism to the ever-changing environmental conditions. Trxs with a pair of cysteine residues at their active site act an important role in disulphide reduction of protein by using NADPH as reducing agent
(Jacquot et al., 2009). This reaction is catalyzed by NADPHdependent thioredoxin reductase (NTR). All the living organisms (including Bacteria, animals, and plants) possess two component NTR/Trx redox systems (Meyer et al., 2005). However, plant chloroplasts have an intricate set of particular Trxs, which additionally utilize a chloroplast specific ferredoxin-dependent thioredoxin reductase (FTR), unlike the other heterotrophic organisms. Hence, rather than the NADPH, the redox regulation of chloroplast is mainly dependent on photosynthetic electron transport chain-reduced ferredoxin in the presence of light. It has been reported that a unique NTR with a Trx domain at its C-terminus (named as NTRC) is utilized in oxygenic photosynthetic organisms and is localized in chloroplasts (Serrato et al., 2004). NTRC is capable of reducing disulphides of the target proteins by using NADPH and hence, it performs as NTR/Trs system in a single polypeptide (Pérez-Ruiz and Cejudo, 2009). After discovery of these results, a new picture appeared according to which both $\mathrm{NADPH}$ and ferredoxin (FD) can be used for maintaining the chloroplast redox homeostasis (Spínola et al., 2008). At night, reduced FD become limiting and NADPH produced from the sugar play as a major source of reducing power and thus, NTRC play an essential role for maintaining the redox homeostasis. It has also been reported that non-green plastids also have the components of FTR/Trx system which suggests that the redox regulation is also occurring in the non-photosynthesizing plant parts (Balmer et al., 2006). The damage in the vital regulatory enzymes of starch synthesis i.e., ADP-glucose pyrophosphorylase, in the NTRC knock out mutant indicated that NTRC might play important role in the redox homeostasis of non-green tissues. The expression analysis of NTRC further showed that it is found in both green chloroplasts and non-green plastids and it could regulate the redox homeostasis in the green and non-green plant parts (Kirchsteiger et al., 2012). Taking together all the recent findings, it can be concluded that redox regulation is an important function of all the plastids (including green and non-green plastids). However, in chloroplasts this depends on light or sugar and in non-green plastids it depends entirely on the NADPH which is generated from the metabolism of sucrose by the oxidative pentose phosphate pathway (Figure 3).

\section{CONTRIBUTION OF MITOCHONDRIA}

Mitochondria also play important role in plant cell redox homeostasis. In the photosynthetic cells, the power house mitochondria are the second key organelle after chloroplasts. Mitochondria have a great contribution toward redox homeostasis during the oxidative reactions operating in mitochondria and peroxisome in the light. Plant mitochondria have specific ETC components which functions in photorespiration process. In leaves, oxygenic photosynthesis certify that mitochondria function in a carbohydrate and oxygen loaded environment. This specific cellular environmental condition ensures the existence of mitochondrial redox signaling and homeostasis. Malate and pyruvate are imported to mitochondria and subsequently oxidized to produce ATP. Another essential function of mitochondria is metabolism of compounds like glutamate and other amino acids, and production of precursors for biosynthetic processes (Ishizaki et al., 2005). In these processes, the expression of the necessary proteins 


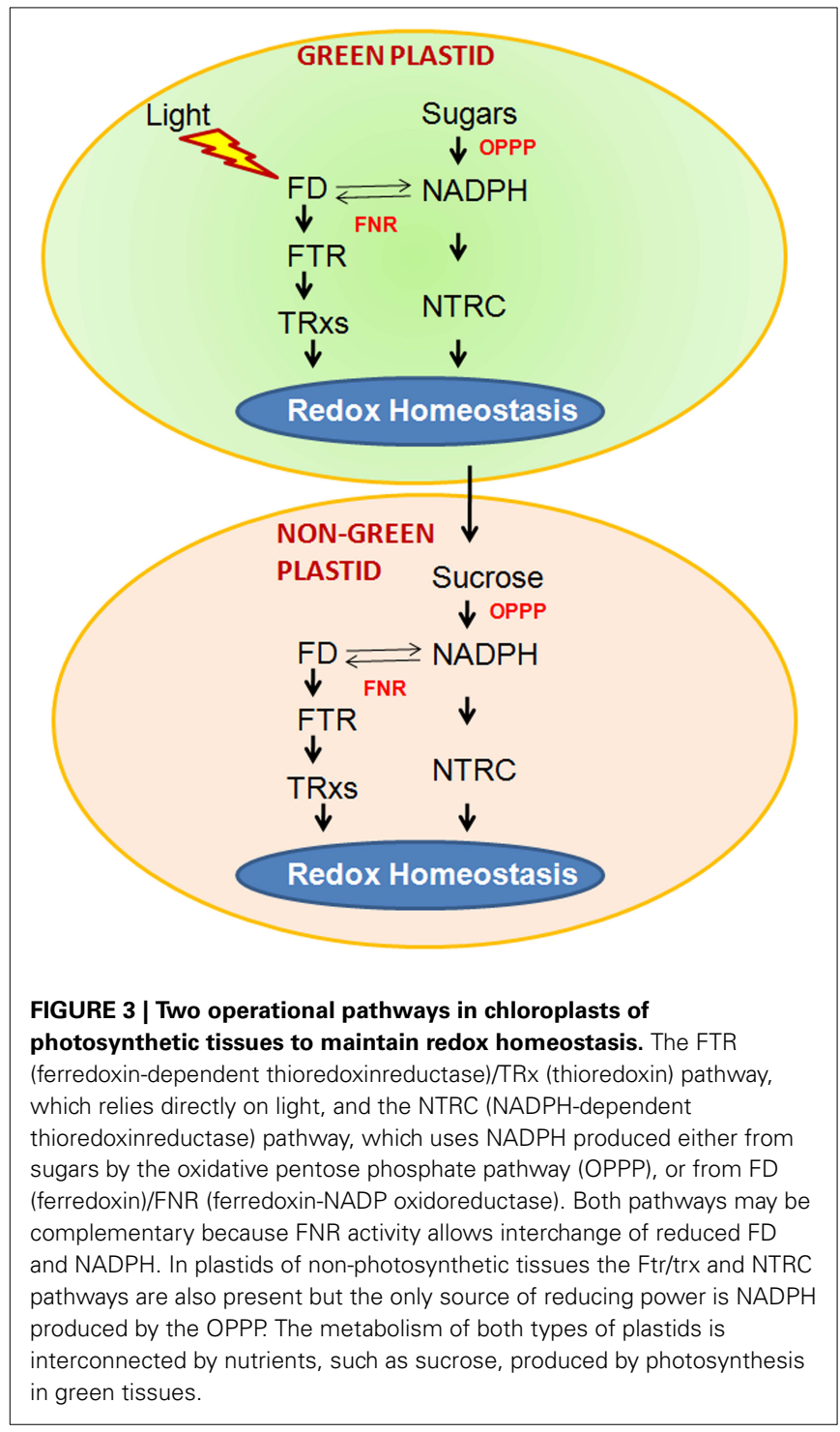

depends upon the developmental stage of the plant and type of the cell. Tricarboxylic acid (TCA) cycle is common to all plant mitochondria but, here, the TCA cycle operates depending on tissue type or environmental factors. Here, APX functions to dissipate electrons without generation of ATP and thus, prevent the formation of ROS during over reduction of the mitochondrial ETC (Vanlerberghe and McIntosh, 1992). Interestingly, APX is a target of redox-modification via the mitochondrial thioredoxin system (Gray et al., 2004). Induction of APX transcription is caused by abiotic stress factors such as low temperature (Vanlerberghe and McIntosh, 1992). By using male sterile mutant tobacco, the role of mitochondria in cellular homeostasis has been shown (Dutilleul et al., 2003). These mutant plants do not have the functional complex I, which is a key complex required for maintaining the redox homeostasis in cell (Noctor et al., 2004). It has also been reported that knockout plants lacking type II peroxiredoxin $\mathrm{F}$ of mitochondria possess a strong phenotype, particularly under stress and when APX is inhibited (Finkemeier et al., 2005). Ultimately, interruption of the TCA cycle by decreasing the quantity of mitochondrial MDH (malate dehdrogenase) had remarkable effect on photosynthesis and plant growth (Nunes-Nesi et al., 2005).

\section{CONTRIBUTION OF PEROXISOME}

Peroxisome is contributing majorly in maintaining cellular redox homeostasis by having the key enzyme CAT inside the peroxisomal boundary. CAT depletes the peroxisomal $\mathrm{H}_{2} \mathrm{O}_{2}$ generated through photorespiratory glycolate oxidase pathway and maintains redox homeostasis of the cell. Plants deficient in CAT have always accumulated high levels of $\mathrm{H}_{2} \mathrm{O}_{2}$. It has been reported that cat 2 mutants grown at relatively low light, possess increased diaminobenzidine staining (Bueso et al., 2007). It has also been reported that cat2 and cat2:cat3 knockout plants contains two folds increase in extractable $\mathrm{H}_{2} \mathrm{O}_{2}$ (Hu et al., 2010). The CATlacking tobacco plants are also more sensitive to diseases as they are not altered in their protein, which is related to pathogenesis, but the tobacco leaves show bleaching due to $\mathrm{H}_{2} \mathrm{O}_{2}$ accumulation in peroxisomes (Chamnongpol et al., 1998). It has also been reported that young leaves are less susceptible than the older leaves, in Cat1 deficient tobacco plants, upon high light exposure (Willekens et al., 1997). Remarkably, double antisense plants deficient in both APX and CAT showed decreased photosynthesis. The reduction of photosynthetic activity is regarded as an approach to avoid the formation of ROS (Rizhsky et al., 2002). Tobacco mutants with increased CAT activity confirmed higher photosynthesis rates under photorespiratory situations than the control, probably because these plants are more tolerant to $\mathrm{O}_{2}$ inhibition of photosynthesis (Zelitch, 1990). Willekens et al. (1997) also reported that Cat1 deficient tobacco plants were unable to maintain ascorbate, particularly glutathione pools in the reduced state when exposed to elevated light conditions. Therefore, peroxisomal localized CAT is an essential enzyme for protecting ascorbate and glutathione pools from oxidation. Additionally, Willekens et al. (1997) also reported that glutathione are the major sulfohydryl component in plants cells, for maintaining the redox homeostasis in light stressed cells. Brisson et al. (1998) have reported that increase in CAT activity reduces the photorespiratory loss of $\mathrm{CO}_{2}$.

\section{CONTRIBUTION OF VACUOLE}

It has been known that the antioxidant system in the vacuolar compartment is comprised of various components of enzymatic and non-enzymatic origin. Apart from the cell wall, Class III peroxidases (POX) are also localized inside the vacuoles and play significant role to quench ROS inside the vacuole, where the secondary metabolites accumulate. Although, the exact function of vacuolar POX is not known, few recent reports show that the vacuolar POX control the level of $\mathrm{H}_{2} \mathrm{O}_{2}$ in photosynthesizing plant cells at the time of oxidation of some vacuolar phenolic substrates with $\mathrm{H}_{2} \mathrm{O}_{2}$ as an electron acceptor (Costa et al., 2008; Brunetti et al., 2011). The presence of POX in the vacuole and the apoplast is a feature of these subcellular compartments known to gather the major part of secondary metabolites which serves as peroxidase substrates (Idanheimo et al., 2014). It has been reported that vacuoles can generate ROS by a mechanism comparable to that in the plasmalemma-apoplast system. This mechanism is 


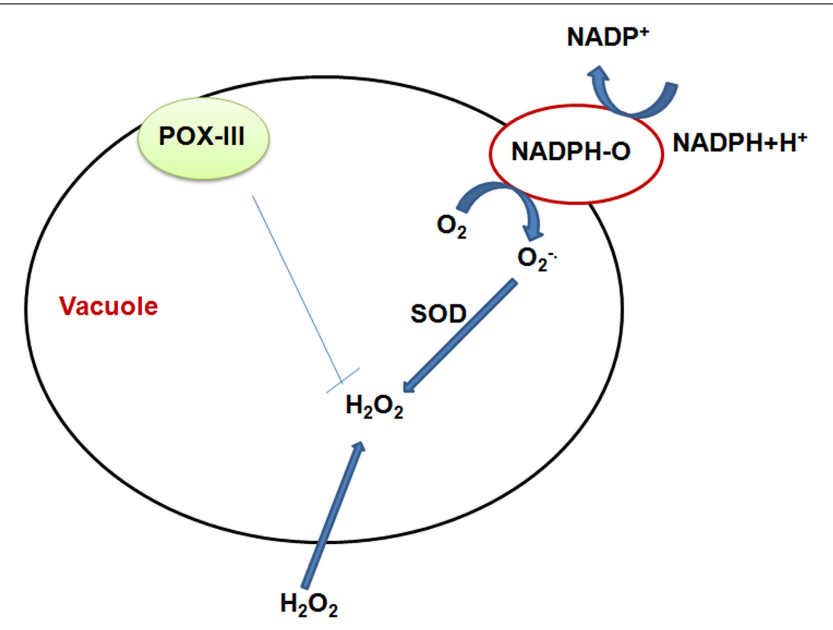

FIGURE 4 | Schematic representation of the vacuolar functioning of redox active enzymes, as well as sugars, namely fructans, acting in cooperation with peroxidases. T-tonoplast; POX-class III peroxidase; NADPH-O-NADPH oxidase; SOD-superoxide dismutase; Functioning in the vacuole of NADPH oxidase and superoxide dismutase is presently a hypothetical possibility, and the validity of this hypothesis is still an open question.

supported by operation of the tonoplast located NADPH oxidase and the vacuolar or tonoplast-surface located superoxide dismutase. These data were acquired from proteomic analysis of the tonoplast membrane proteins and biochemical recognition of the enzymes (Shi et al., 2007; Whiteman et al., 2008; Pradedova et al., 2011). However, the convincing and direct experimental confirmation for functioning of such enzymes in the vacuolar compartment is not yet reported. The presence of superoxide producing NADPH oxidase in membranes of animal phagocytes and lysosomes cannot be taken as enough evidence for the presence of a similar enzyme in the tonoplast of plant cell. The schematic representation of mechanism of ROS quenching involving vacuolar enzymes is shown in Figure 4.

\section{CONTRIBUTION OF CELL WALL AND PLASMA MEMBRANE}

Apart from the major cell organelles, cell wall also plays crucial role in maintaining redox balance in the cell. Similar to the other organelles, oxidative burst also occurs in the plant cell wall where, molecular oxygen is reduced to $\mathrm{O}_{2}^{-}$and then undergoes spontaneous dismutation at a higher rate at acidic $\mathrm{pH}$ (O'Brien et al., 2012). Class III POX present in the cell wall are able to oxidize $\mathrm{NADH}$ and catalyze the formation of $\mathrm{O}_{2}^{-}$. The cell wall oxidases catalyze the oxidation of $\mathrm{NADH}$ to $\mathrm{NAD}^{+}$, which in turn reduces oxygen to superoxide. This superoxide consequently dismutated to produce $\mathrm{H}_{2} \mathrm{O}_{2}$ andO 2 (Bhattachrjee, 2005; O'Brien et al., 2012). Additionally, amine oxidases and oxalate oxidases have been proposed to generate $\mathrm{H}_{2} \mathrm{O}_{2}$ in the apoplast (Munné-Bosch et al., 2013). NADPH oxidase present in cell membrane is another source of $\mathrm{H}_{2} \mathrm{O}_{2}$ for oxidative burst ( $\mathrm{O}$ 'Brien et al., 2012). Aluminum, a soil pollutant, is also responsible for oxidative burst through activating the cell wall-NADH peroxidase and/or plasma membrane-associated NADPH oxidase (Achary et al., 2012). However, it is evident that presence of SOD in the cell wall is responsible for the efficient scavenging of the $\mathrm{O}_{2}^{-}$(Apel and Hirt, 2004). It has also been reported that along with class III POX, APX is also present in cell wall and plasma membrane which is responsible for depletion of $\mathrm{H}_{2} \mathrm{O}_{2}$ and helps in maintaining cellular redox homeostasis (Apel and Hirt, 2004; O'Brien et al., 2012).

\section{CROSS TALK AMONG CELLULAR ORGANELLES}

The peroxisomal extension, named peroxules, can expand over the chloroplastic exterior and curl around it, in a very quick manner and connect with other peroxisomes (Sinclair et al., 2009). Morphology of peroxisome can modify under stress situations which induce a quick key between spherical motile organelles with extensive tubular-beaded shape with extended peroxules (Sinclair et al., 2009). Stromules are stroma-filled tubules present in chloroplasts, consisting of thin extensions of the stroma (Hanson and Sattarzadeh, 2008) and these can often join together and have been shown to enter into channels of the nucleus (Kwok and Hanson, 2004). Chloroplasts, peroxisome and mitochondria have high rates of ROS metabolism which vary with the changing environmental conditions. Close interactions between chloroplast, peroxisomes and mitochondria could enhance cellular metabolic synchronization under stress situations and contribute to plant stress acceptance/tolerance (Rivero et al., 2009). Furthermore, increase of mitochondria and peroxisomes at the diffusion/penetration site of a fungus has been shown which probably occur for detoxification of the ROS at the infected site of the fungus Erysiphe cichoracearum (Koh et al., 2005). Form the above studies, it is quite convincing to state that that the cellular organellar crosstalk play significant role in cell signaling, avoiding stress situation and maintaining the cell redox homeostasis.

\section{DEVELOPMENT OF TRANSGENIC PLANTS TOLERANT TO ABIOTIC STRESS BY ENHANCING ROS DEFENSE MECHANISMS}

In past, researchers have developed several transgenic plants by manipulating various genes involved in enzymatic and nonenzymatic ROS scavenging mechanisms which have shown increased tolerance to abiotic stresses (Table 1).

Over-expression of genes encoding ROS-scavenging enzymes such as SOD (Prashanth et al., 2008), CAT (Al-Taweel et al., 2007), APX (Kim et al., 2008), MDAR (Etrayeb et al., 2007), DHAR (Ushimaru et al., 2006), GR (Kornyeyev et al., 2003) and GPX (Gaber et al., 2006) in various plants isolated from same or different organisms were shown to possess higher tolerance to one or more abiotic stresses by minimizing the oxidative damage. Complete neutralization of ROS molecules involves more than one enzymes localized in same or different sub cellular compartments of cell. Transgenic Cassava (Manihot esculenta Crantz) has also shown the increased level of other important ROS scavenging enzymes such as MDR, DHAR, and GR.

Similarly, overexpression of critical enzymes involved in the biosynthetic pathway of antioxidants play a significant role in combating different abiotic stresses. Overexpression of P5CS (Yamada et al., 2005; Vendruscolo et al., 2007), a key enzyme for proline biosynthesis leads to increased tolerance to drought in transgenic plants. Liu et al. (2008) generated tobacco transgenic 
Table 1 | Representative reports for raising transgenic plants by overexpressing enzymes involved in ROS scavenging, which show improved tolerance to various abiotic stresses.

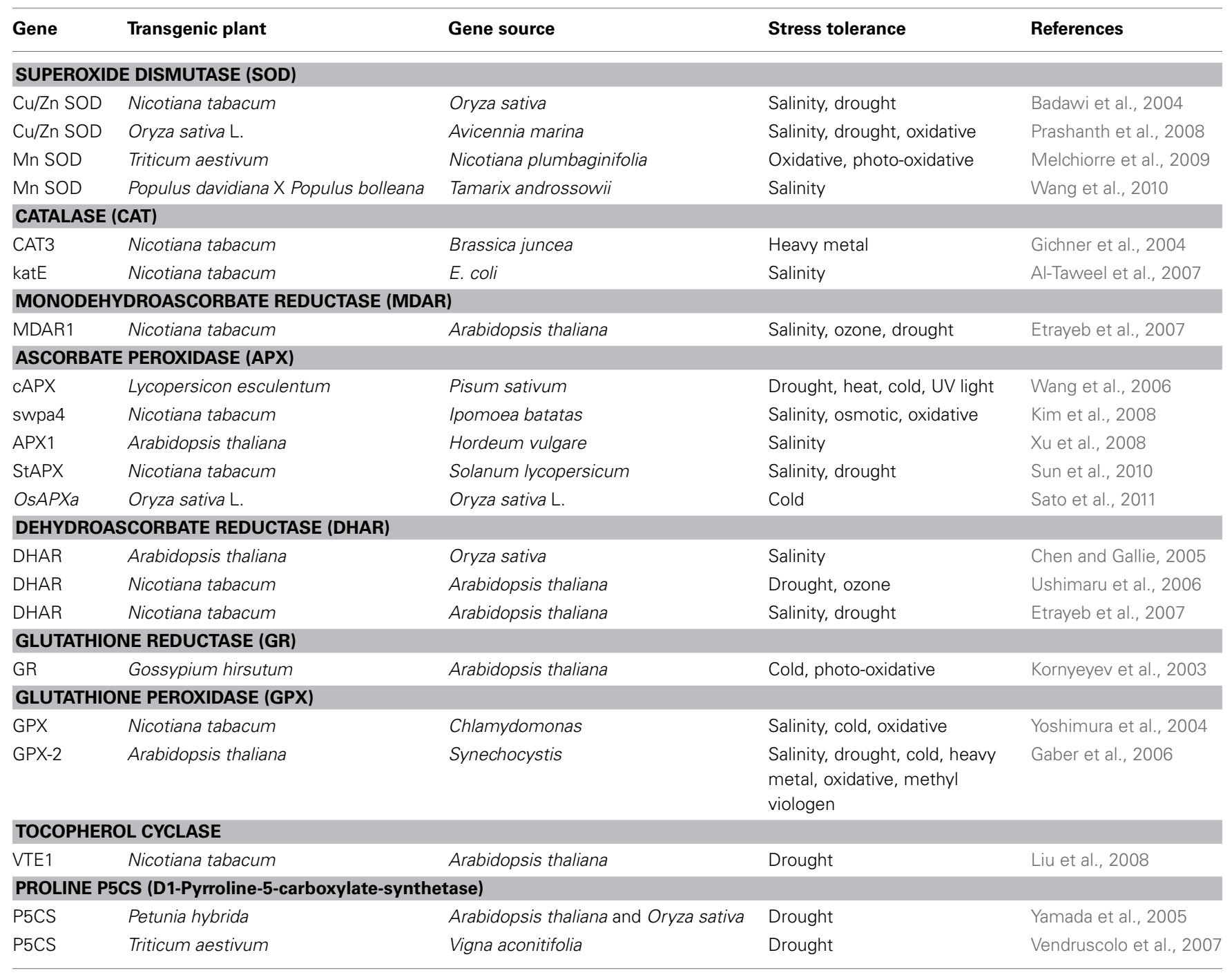

plants by overexpressing VTE1 gene, encoding tocopherol cyclase (VTE1), an important enzyme involved in tocopherol biosynthesis. They have showed that the VTE1 overexpressing plants have higher tolerance to drought. Increased accumulation of another important antioxidant -ascorbic acid in AtERF98 TF overexpressing transgenic arabidopsis, showed increased tolerance to salinity (Zhang et al., 2012).

Apart from ROS-scavenging enzymes and non-enzymatic antioxidants, over-expressing ROS-responsive signaling and regulatory genes also responsible for stress tolerance in plants. The regulatory genes which regulate a large set of genes involved in acclimation mechanisms, including ROS-scavenging enzymes proved beneficial in enhancing tolerance to abiotic stresses such as drought, salinity, oxidative, cold and heavy metal stress. In Arabidopsis, over-expression of mitogen-activated kinase kinase 1 (MKK1) enhanced the activity of MAPK cascade, which is also activated by ROS (Teige et al., 2004; Wrzaczek et al., 2013) leads to increased tolerance to abiotic stresses by controlling stress-associated ROS levels under abiotic stress (Xing et al., 2008). Likewise, over-expression of transcription factors (Zat12 or JERF3, Zat10) control the expression of various ROSscavenging genes encoding enzymes showed higher tolerance to salt, drought or osmotic stresses (Sakamoto et al., 2004; Davletova et al., 2005). Rai et al. (2013) have reported that overexpression of AtDREB1A/CBF3 of Arabidopsis under the control of stress inducible promoter (rd29A) in tomato (cv. Kashi Vishesh) showed higher accumulation of ROS scavenging enzymes and antioxidants with greater tolerance to drought-induced oxidative stress.

It has been established that the transgenic plants produced through gene pyramiding or co-expression of several antioxidant genes could able to give better stress tolerance than the plants overexpressing a single antioxidant gene (Table 2). It has been reported that co-expression of Mn-SOD and APX could able enhance multiple abiotic stress tolerance in Nicotiana tabacum. Co-expression of maize $\mathrm{ZmCu} / \mathrm{ZnSOD}$ and $\mathrm{ZmCAT}$ 
Table 2 | Representative reports for raising transgenic plants by co-expressing enzymes involved in ROS scavenging, which show improved tolerance to various abiotic stresses.

\begin{tabular}{|c|c|c|c|c|}
\hline Genes & Transgenic plant & Gene source & Stress tolerance & References \\
\hline Mn SOD + APX & Festuca arundinacea & Nicotiana tabacum & Multiple abiotic stresses, oxidative & Lee et al., 2007 \\
\hline $\mathrm{Cu} / \mathrm{Zn} \mathrm{SOD}+\mathrm{CAT}$ & Brassica campestris & Zea maize & Salinity, $\mathrm{SO}_{2}$ & Tseng et al., 2007 \\
\hline cytAPX + cytSOD & Prunus domestica & $\begin{array}{l}\text { cytsod from spinach and } \\
\text { cytapx from pea }\end{array}$ & Salinity, oxidative & Diaz-Vivancos et al., 2013 \\
\hline MeAPX2 + MeCu/ZnSOD & Manihot esculenta Crantz & Manihot esculenta Crantz & Chilling, oxidative & Xu et al., 2014 \\
\hline
\end{tabular}

showed higher photosynthetic efficiency and salinity tolerance ability of transgenic cabbage (Brassica campestris L.) better than the independent $\mathrm{ZmCu} / \mathrm{ZnSOD}$ and $\mathrm{ZmCAT}$ transgenic plant (Tseng et al., 2007). Likewise, co-expression of MeAPX2 and $\mathrm{MeCu} / \mathrm{ZnSOD}$ in cassava (Manihot esculenta Crantz) showed higher tolerance to MV mediated $\mathrm{H}_{2} \mathrm{O}_{2}$ stress as well as two fold tolerance to chilling stress as compare to the wild type plants (Xu et al., 2014).

\section{CONCLUSION AND FUTURE PROSPECTS}

Normally, ROS are generated by metabolic activity of the plants and act as signaling molecules for activating plant metabolic pathway. However, under environmental stresses, generation of ROS increase in different compartments of the cell such as chloroplast, peroxisomes and mitochondria. Higher accumulation of ROS leads to oxidative stress in plant causing damage to the cell membranes (lipid peroxidation) and biomolecules like nucleic acid, protein and lipid by oxidative damage. To combat the harmful effect of increased ROS accumulation, plants are equipped with effective ROS scavenging mechanisms. Plants have evolved two types of scavenging tools (i) scavenging enzymes such as SOD, CAT, MDAR, dehydroascorbate reductase (DHAR), GR and glutathione peroxidase (GP) and (ii) antioxidant molecules like ascorbic acid, $\alpha$-tocopherols, glutathione, proline, flavonoids and carotenoids. ROS are key signaling molecules interacting with each other and with other cellular antioxidant systems to maintain proper balance between various cellular metabolic pathways, which get disrupted under unfavorable environments. Therefore, it is not the ROS, but their concentration in cell which decides their good or bad effect on plant. A lot of information about the ROS generation, role of free radicals in intra cellular communication and their effective scavenging have been accessible, but there are gaps in our understanding of complete ROS scavenging and signaling pathway. Future research in this area will be useful for designing the strategy to achieve the potential yield under unfavorable environments. Although, through transgenic technology of ROS scavenging components, abiotic biotic stress tolerance in various crop plants has been improved to some extent, this needs to be improved further in future by gene pyramiding to achieve the near potential yield of crops under rapidly changing climate.

\section{ACKNOWLEDGMENTS}

The authors gratefully acknowledge financial support from University Grant Commission (through resource network program to JNU and Dr D. S. Kothari fellowship to PD) and Department of Biotechnology, Government of India.

\section{REFERENCES}

Achary, V. M. M., Parinandi, N. L., and Panda, B. B. (2012). Aluminum induces oxidative burst, cell wall NADH peroxidase activity, and DNA damage in root cells of Allium cepa L. Environ. Mol. Mutagen. 53, 550-560. doi: 10.1002/em.21719

Al-Taweel, K., Iwaki, T., Yabuta, Y., Shigeoka, S., Murata, N., and Wadano, A. (2007). A bacterial transgene for catalase protects translation of $\mathrm{d} 1$ protein during exposure of salt-stressed tobacco leaves to strong light. Plant Physiol. 145, 258-265. doi: 10.1104/pp.107.101733

Andreev, I. M. (2012). Role of the vacuole in the redox homeostasis of plant cells. Russ. J. Plant Physiol. 59, 611-617. doi: 10.1134/S1021443712050032

Apel, K., and Hirt, H. (2004). Reactive oxygen species: metabolism, oxidative stress, and signal transduction. Annu. Rev. Plant Biol. 55, 373-399. doi: 10.1146/annurev.arplant.55.031903.141701

Aung-Htut, M. T., Ayer, A., Breitenbach, M., and Dawes, I. W. (2012). "Oxidative stresses and ageing," in Aging Research in Yeast, eds M. Breitenbach, S. M. Jazwinski, and P. Laun, (Dordrecht: Springer), 13-54.

Badawi, G. H., Yamauchi, Y., Shimada, E., Sasaki, R., Kawano, N., and Tanaka, K. (2004). Enhanced tolerance to salt stress and water deficit by overexpressing superoxide dismutase in tobacco (Nicotiana tabacum) chloroplasts. Plant Sci. 166, 919-928. doi: 10.1016/j.plantsci.2003.12.007

Bailey-Serres, J., and Voesenek, L. A. C. J. (2008). Flooding stress: acclimations and genetic diversity. Annu. Rev. Plant Biol. 59, 313-339. doi: 10.1146/annurev.arplant.59.032607.092752

Balmer, Y., Vensel, W. H., Cai, N., Manieri, W., Schürmann, P., Hurkman, W. J., et al. (2006). A complete ferredoxin/ thioredoxin system regulates fundamental processes in amyloplasts. Proc. Natl. Acad. Sci. U.S.A. 103, 2988-2993. doi: 10.1073/pnas.0511040103

Bhattachrjee, S. (2005). Reactive oxygen species and oxidative burst: roles in stress, senescence and signal transduction in plant. Curr. Sci. 89, 1113-1121.

Borisjuk, L., and Rolletschesk, H. (2008). The oxygen status of the developing seed. New Phytol. 182, 17-30. doi: 10.1111/j.1469-8137.2008.02752.x

Brisson, L. F., Zelitch, I., and Havir, E. A. (1998). Manipulation of catalase levels produces altered photosynthesis in transgenic tobacco plants. Plant Physiol. 116, 259-269. doi: 10.1104/pp.116.1.259

Brunetti, F. A., Ferdinando, D. M., Ferrini, F., and Tattini, M. (2011). Stress induced flavonoid biosynthesis and the antioxidant machinery of plants. Plant Signal. Behav. 6, 709-711. doi: 10.4161/psb.6.5.15069

Bueso, E., Alejandro, S., Carbonell, P., Perez-Amador, M. A., Fayos, J., Belles, J. M., et al. (2007). The lithium tolerance of the Arabidopsis cat 2 mutant reveals a cross talk between oxidative stress and ethylene. Plant J. 52, 1052-1065. doi: 10.1111/j.1365-313X.2007.03305.x

Chamnongpol, S., Willekens, H., Moder, W., Langebartels, C., Sondermann, H., Van Montagu, M., et al. (1998). Defense activation and enhanced pathogen tolerance induced by $\mathrm{H}_{2} \mathrm{O}_{2}$ in transgenic plants. Proc. Natl. Acad. Sci. U.S.A. 95, 5818-5823. doi: 10.1073/pnas.95.10.5818

Chen, Z., and Gallie, D. R. (2005). Increasing tolerance to ozone by elevating folia ascorbic acid confers greater protection against ozone than increasing avoidance. Plant Physiol. 138, 1673-1689. doi: 10.1104/pp.105.062000

Chiu, J., and Dawes, I. W. (2012). Redox control of cell proliferation. Trends Cell Biol. 22, 592-601. doi: 10.1016/j.tcb.2012.08.002

Costa, M. M. R., Hilliou, F., Duarte, P., Pereira, L. G., Almeida, I., Leech, M., et al. (2008). Molecular cloning and characterization of a vacuolar class III peroxodase involved in the metabolism of anticancer alkaloid in Catharanthus roseus. Plant Physiol. 146, 403-417. doi: 10.1104/pp.107.107060 
Cvetkovska, M., Rampitsch, C., Bykova, N., and Xing, T. (2005). Genomic analysis of MAP kinase cascades in Arabidopsis defense responses. Plant Mol. Biol. Rep. 23, 331-343. doi: 10.1007/BF02788882

Davletova, S., Schlauch, K., Coutu, J., and Mittler, R. (2005). The zinc-finger protein Zat12 plays a central role in reactive oxygen and abiotic stress signaling in Arabidopsis. Plant Physiol. 139, 847-856. doi: 10.1104/pp.105.068254

Diaz-Vivancos, P., Faize, M., Barba-Espin, G., Faize, L., Petri, C., Hernández, J. A., et al. (2013). Ectopic expression of cytosolic superoxide dismutase and ascorbate peroxidase leads to salt stress tolerance in transgenic plums. Plant Biotechnol. J. 11, 976-985. doi: 10.1111/pbi.12090

Dutilleul, C., Garmier, M., Noctor, G., Mathieu, C., Chetrit, P., Foyer, C. H., et al. (2003). Leaf mitochondria modulate whole cell redox homeostasis, set antioxidant capacity, and determine stress resistance through altered signalling and diurnal regulation. Plant Cell. 15, 1212-1226. doi: 10.1105/tpc. 009464

Etrayeb, A. E., Kawano, N., Badawi, G. H., Kaminaka, H., Sanekata, T., Shibahara, T., et al. (2007). Overexpression of monodehydroascorbate reductase in transgenic tobacco confers enhanced tolerance to ozone, salt and polyethylene glycol stresses. Planta 225, 1255-1264. doi: 10.1007/s00425-006-0417-7

Ferrández, J., González, M., and Cejudo, F. J. (2012). Chloroplast redox homeostasis is essential for lateral root formation in Arabidopsis. Plant Signal. Behav. 7 , 1177-1179. doi: 10.4161/psb.21001

Finkemeier, I., Goodman, M., Lamkemeyer, P., Kandlbinder, A., Sweetlove, L. J., and Dietz, K. J. (2005). The mitochondrial type II peroxiredoxin F is essential for redox homeostasis and root growth of Arabidopsis thaliana under stress. J. Biol. Chem. 280, 12168-12180. doi: 10.1074/jbc.M413189200

Foyer, C. H., and Noctor, G. (2005). Redox homeostasis and antioxidant signaling: a metabolic interface between stress perception and physiological responses. Plant Cell. 17, 1866-1875. doi: 10.1105/tpc.105.033589

Gaber, A., Yoshimura, K., Yamamoto, T., Yabuta, Y., Takeda, T., Miyasaka, H., et al. (2006). Glutathione peroxidase-like protein of Synechocystis PCC 6803 confers tolerance to oxidative and environmental stresses in transgenic Arabidopsis. Physiol. Plant. 128, 251-262. doi: 10.1111/j.1399-3054.2006.00730.x

Ghosh, A., Pareek, A., Sopory, S. K., and Singla-Pareek, S. L. (2014). A glutathione responsive rice glyoxalase II, OsGLYII-2, functions in salinity adaptation by maintaining better photosynthesis efficiency and anti-oxidant pool. Plant J. 80, 93-105. doi: 10.1111/tpj.12621

Gichner, T., Patkova, Z., Szakova, J., and Demnerova, K. (2004). Cadmium induces DNA damages in tobacco roots, but no DNA damage, somatic mutations or homologous recombinations in tobacco leaves. Mutat. Res. 559, 49-57. doi: 10.1016/j.mrgentox.2003.12.008

Gray, M. W., Lang, B. F., and Burger, G. (2004). Mitochondria of protists. Annu. Rev. Genet. 38, 477-524. doi: 10.1146/annurev.genet.37.110801.142526

Hanson, M. R., and Sattarzadeh, A. (2008). Dynamic morphology of plastids and stromules in angiosperm plants. Plant Cell Environ. 31, 646-657. doi: 10.1111/j.1365-3040.2007.01768.x

Hu, Y. Q., Liu, S., Yuan, H. M., Li, J., Yan, D. W., Zhang, J. F., et al. (2010). Functional comparison of catalase genes in the elimination of photorespiratory $\mathrm{H} 2 \mathrm{O} 2$ using promoter- and 3 -untranslated region exchange experiments in the Arabidopsis cat2 photorespiratory mutant. Plant Cell Environ. 33, 1656-1670. doi: 10.1111/j.1365-3040.2010.02171.x

Idanheimo, N., Gauthier, A., Salojarvi, J., Siligato, R., Brosché, M., Kollist, H., et al. (2014). The Arabidopsis thaliana cysteine-rich receptor-like Kinases CRK6 and CRK7 protect against apoplastic oxidative stress. Biochem. Biophys. Res. Commun. 445, 457-462. doi: 10.1016/j.bbrc.2014.02.013

Ishizaki, K., Larson, T. R., Schauer, N., Fernie, A. R., Graham, I. A., and Leaver, C. J. (2005). The critical role of Arabidopsis electron-transfer flavoprotein:ubiquinone oxidoreductase during dark-induced starvation. Plant Cell. 17, 2587-2600. doi: 10.1105/tpc.105.035162

Jacquot, J.-P., Eklund, H., Rouhier, N., and Schürmann, P. (2009). Structural and evolutionary aspects of thioredoxinreductases in photosynthetic organisms. Trends Plant Sci. 14, 336-343. doi: 10.1016/j.tplants.2009.03.005

Kangasjarvi, S., and Kangasjarvi, J. (2014). Towards understanding extracellular ROS sensory and signaling systems in plants. Adv. Bot. doi: $10.1155 / 2014 / 538946$

Kanwischer, M., Porfirova, S., Bergmuller, E., and Dormann, P. (2005). Alterations in tocopherol cyclase activity in transgenic and mutant plants of Arabidopsis affect tocopherol content, tocopherol composition, and oxidative stress. Plant Physiol. 137, 713-723. doi: 10.1104/pp.104.054908
Khanna-Chopra, R., Nutan, K. K., and Pareek, A. (2013). "Regulation of leaf senescence: role of reactive oxygen species," in Plastid Development in Leaves during Growth and Senescence, eds B. Biswal, K. Krupinska, and U. C. Biswal (Dordrecht: Springer), 393-416.

Kiffin, R., Bandyopadhyay, U., and Cuervo, A. M. (2006). Oxidative stress and autophagy. Antioxid. Redox Signal. 8, 152-162. doi: 10.1089/ars.2006.8.152

Kim, Y. H., Kim, C. Y., Song, W. K., Park, D. S., Kwon, S. Y., Lee, H. S., et al. (2008). Overexpression of sweetpotato swpa4 peroxidase results in increased hydrogen peroxide production and enhances stress tolerance in tobacco. Planta 227, 867-881. doi: 10.1007/s00425-007-0663-3

Kirchsteiger, K., Ferrández, J., Pascual, M. B., González, M., and Cejudo, F. J. (2012). NADPH thioredoxin reductase $\mathrm{C}$ is localized in plastids of photosynthetic and nonphotosynthetic tissues and is involved in lateral root formation in Arabidopsis. Plant Cell. 24, 1534-1548. doi: 10.1105/tpc.111.092304

Koh, S., André, A., Edwards, H., Ehrhardt, D., and Somerville, S. (2005). Arabidopsis thaliana subcellular responses to compatible Erysiphe cichoracearum infections. Plant J. 44, 516-529. doi: 10.1111/j.1365-313X.2005.02545.x

Kornyeyev, D., Logan, B. A., Payton, P., Allen, R. D., and Holaday, A. S. (2003). Elevated chloroplastic glutathione reductase activities decrease chillinginduced photoinhibition by increasing rates of photochemistry, but not thermal energy dissipation, in transgenic cotton. Funct. Plant Biol. 30, 101-110. doi: 10.1071/FP02144

Kumar, G., Kushwaha, H. R., Panjabi-Sabharwal, V., Kumari, S., Joshi, R., Karan, R., et al. (2012). Clustered metallothionein genes are co-regulated in rice and ectopic expression of OsMT1e-P confers multiple abiotic stress tolerance in tobacco via ROS scavenging. BMC Plant Biol. 12, 107-121. doi: 10.1186/14712229-12-107

Kwok, E. Y., and Hanson, M. R. (2004). Plastids and stromules interact with the nucleus and cell membrane in vascular plants. Plant Cell Rep. 23, 188-195. doi: 10.1007/s00299-004-0824-9

Kwon, S. J., Choi, E. Y., and Choi, Y. J. (2006). Proteomics studies of post-translational modifications in plants. J. Exp. Bot. 57, 1547-1551. doi: 10.1093/jxb/erj137

Lázaro, J. J., Jiménez, A., Camejo, D., Iglesias-Baena, I., Martí, M. C., Lázaro-Payo, A., et al. (2013). Dissecting the integrative antioxidant and redox systems in plant mitochondria. Effect of stress and S-nitrosylation. Front. Plant Sci. 4, 1-20. doi: 10.3389/fpls.2013.00460

Lee, S. H., Ahsan, N., Lee, K. W., Kim, D. H., Lee, D. G., Kwak, S. S., et al. (2007). Simultaneous overexpression of both $\mathrm{CuZn}$ superoxide dismutase and ascorbate peroxidase in transgenic tall fescue plants confers increased tolerance to a wide range of abiotic stresses. J. plant Physiol. 164, 1626-1638. doi: 10.1016/j.jplph.2007.01.003

Li, J. M., and Jin, H. (2007). Regulation of brassinosteroid signalling, Trends Plant Sci. 12, 37-41. doi: 10.1016/j.tplants.2006.11.002

Liu, X., Hua, X., Guo, J., Qi, D., Wang, L., Liu, Z., et al. (2008). Enhanced tolerance to drought stress in transgenic tobacco plants overexpressing VTE1 for increased tocopherol production from Arabidopsis thaliana. Biotechnol. Lett. 30, 1275-1280. doi: 10.1007/s10529-008-9672-y

Melchiorre, M., Robert, G., Trippi, V., Racca, R., and Lascano, H. R. (2009) Superoxide dismutase and glutathione reductase overexpression in wheat protoplast: photooxidative stress tolerance and changes in cellular redox state. Plant Growth Regul. 57, 57-68. doi: 10.1007/s10725-008-9322-3

Meyer, Y., Reichheld, J. P., and Vignols, F. (2005). Thioredoxins in Arabidopsis and other plants. Photosynth. Res. 86, 419-433. doi: 10.1007/s11120-0055220-y

Miao, Y., Laun, T. M., Smykowski, A., and Zentgraf, U. (2007). Arabidopsis MEKK1 can take a short cut: it can directly interact with senescence-related WRKY53 transcription factor on the protein level and can bind to its promoter. Plant Mol. Biol. 65, 63-76. doi: 10.1007/s11103-007-9198-Z

Munné-Bosch, S., Queval, G., and Foyer, C. H. (2013). The impact of global change factors on redox signaling underpinning stress tolerance. Plant Physiol. 161, 5-19. doi: 10.1104/pp.112.205690

Nakashima, K., and Yamaguchi-Shinozaki, K. (2006). Regulons involved in osmotic stress-responsive and cold stress-responsive gene expression in plants. Physiol. Plant. 126, 62-71. doi: 10.1111/j.1399-3054.2005.00592.x

Noctor, G., Dutilleul, C., De Paepe, R., and Foyer, C. H. (2004). Use of mitochondrial electron transport mutants to evaluate the effects of redox state on photosynthetic stress tolerance anad the integration of carbon/nitrogen metabolism. J. Exp. Bot. 55, 49-57. doi: 10.1093/jxb/erh021 
Nunes-Nesi, A., Carrari, F., Lytovchenko, A., Smith, A. M. O., Ehlers Loureiro, M., Ratcliffe, R. G., et al. (2005). Enhanced photosynthetic performance and growth as a consequence of decreasing mitochondrial malate dehydrogenase activity in transgenic tomato plants. Plant Physiol. 137, 611-622. doi: 10.1104/pp.104.055566

O'Brien, J. A., Daudi, A., Butt, V. S., and Bolwell, G. P. (2012). Reactive oxygen species and their role in plant defence and cell wall metabolism. Planta 236, 765-779. doi: 10.1007/s00425-012-1696-9

Panda, B. B., Achary, V. M. M., Mahanty, S., and Panda, K. K. (2013). "Plant adaptation to abiotic and genotoxic stress: relevance to climate change and evolution," in Climate Change and Abiotic Stress Tolerance, Vol. 10, eds N. Tuteja and S. S. Gill (Weinheim: Wiley Wiley-VCH Verlag GmbH \& Co.), 251-294. doi: 10.1002/9783527675265.ch10

Pérez-Ruiz, J. M., and Cejudo, F. J. (2009). A proposed reaction mechanism for rice $\mathrm{NADPH}$ thioredoxinreductase $\mathrm{C}$, an enzyme with protein disulfide reductase activity. FEBS Lett. 583, 1399-1402. doi: 10.1016/j.febslet.2009.03.067

Pfannschmidt, T., Schutze, K., and Fey, V. (2003). Chloroplast redox control of nuclear gene expression-A new class of plastid signals in interorganellar communication. Antioxid. Redox Signal. 5, 95-101. doi: $10.1089 / 152308603321223586$

Pradedova, E. V., Isheeva, O. D., and Salyaev, R. K. (2011). Antioxidant defense enzymes in cell vacuoles of red beet roots. Russ. J. Plant Physiol. 58, 36-44. doi: 10.1134/S1021443711010110

Prashanth, S. R., Sadhasivam, V., and Parida, A. (2008). Over expression of cytosolic copper/zinc superoxide dismutase from a mangrove plant Avicennia marina in indica Rice var Pusa Basmati-1 confers abiotic stress tolerance. Transgenic Res. 17, 281-291. doi: 10.1007/s11248-007-9099-6

Rai, G. K., Rai, N. P., Rathaur, S., Kumar, S., and Singh, M. (2013). Expression of rd29A::AtDREB1A/CBF3 in tomato alleviates drought-induced oxidative stress by regulating key enzymatic and non-enzymatic antioxidants. Plant Physiol. Biochem. 69, 90-100. doi: 10.1016/j.plaphy.2013.05.002

Rio, L. A. D., Sandalio, L. M., and Corpas, F. J. (2006). Reactive oxygen species and reactive nitrogen species in peroxisomes. Production, scavenging and role in cell signaling. Plant Physiol. 141, 330-335. doi: 10.1104/pp.106.078204

Rivero, R. M., Shulaev, V., and Blumwald, E. (2009). Cytokinin-dependent photorespiration and the protection of photosynthesis during water deficit. Plant Physiol. 150, 1530-1540. doi: 10.1104/pp.109.139378

Rizhsky, L., Hallak-Herr, E., Van Breusegem, F., Rachmilevitch, S., Rodermel, S., Inze, D., et al. (2002). Double antisense plants lacking ascorbate peroxidase and catalase are less sensitive to oxidative stress than single antisense plants lacking ascorbate peroxidase or catalase. Plant J. 32, 329-342. doi: 10.1046/j.1365-313X. 2002.01427

Sakamoto, H., Maruyama, K., Sakuma, Y., Meshi, T., Iwabuchi, M., Shinozaki, K., et al. (2004). Arabidopsis Cys2/His2-type zinc-finger proteins function as transcription repressors under drought, cold, and high-salinity stress conditions. Plant Physiol. 136, 2734-2746. doi: 10.1104/pp.104.046599

Sato, Y., Masuta, Y., Saito, K., Murayama, S., and Ozawa, K. (2011). Enhanced chilling tolerance at the booting stage in rice by transgenic overexpression of the ascorbate peroxidase gene, OsAPXa. Plant Cell Rep. 30, 299-406. doi: 10.1007/s00299-010-0985-7

Serrato, A. J., Pérez-Ruiz, J. M., Spínola, M. C., and Cejudo, F. J. (2004). A novel NADPH thioredoxinreductase, localized in the chloroplast, which deficiency causes hypersensitivity to abiotic stress in Arabidopsis thaliana. J. Biol. Chem. 279, 43821-43827. doi: 10.1074/jbc.M404696200

Shao, H. B., Chu, L. Y., Zhao, C. X., Guo, Q. J., Liu, X. A., and Ribaut, J. M. (2006). Plant gene regulatory net work system under abiotic stress. Acta Biol. Sezeged. 50, 1-9.

Shi, Q. H., Wang, X. F., and Wei, M. (2007). Nitric oxide modulates the metabolism of plasma membrane and tonoplast in cucumber roots, Acta Hortic. 761, 275-282.

Sinclair, A. M., Trobacher, C. P., Mathur, N., Greenwood, J. S., and Mathur, J. (2009). Peroxule extension over ER-defined paths constitutes a rapid subcellular response to hydroxyl stress. Plant J. 59, 231-242. doi: 10.1111/j.1365313X.2009.03863.x

Spínola, M. C., Pérez-Ruiz, J. M., Pulido, P., Kirchsteiger, K., Guinea, M., González, M. C., et al. (2008). NTRC new ways of using NADPH in the chloroplast. Physiol. Plant. 133, 516-524. doi: 10.1111/j.1399-3054.2008.01088.x

Sun, W. H., Duan, M., Shu, D. F., Yang, S., and Meng, Q. W. (2010). Overexpression of StAPX in tobacco improves seed germination and increases early seedling tolerance to salinity and osmotic stresses. Plant Cell Rep. 29, 917-926. doi: 10.1007/s00299-010-0878-9

Teige, M., Scheikl, E., Eulgem, T., Dóczi, F., Ichimura, K., Shinozaki, K., et al. (2004). The MKK2 pathway mediates cold and salt stress signaling in Arabidopsis. Mol. Cell. 15, 141-152. doi: 10.1016/j.molcel.2004. 06.023

Terman, A., and Brunk, U. T. (2006). Oxidative stress, accumulation of biological 'Garbage' and aging. Antioxid. Redox Signal. 8, 197-204. doi: 10.1089/ars.2006.8.197

Thorpe, G. W., Fong, C. S., Alic, N., Higgins, V. J., and Dawes, I. W. (2004). Cells have distinct mechanisms to maintain protection against different reactive oxygen species: oxidative-stress-response genes. Proc. Natl. Acad. Sci. U.S.A. 101, 6564-6569. doi: 10.1073/pnas.0305888101

Tokunaga, T., Miyahara, K., Tabata, K., and Esaka, M. (2005). Generation and properties of ascorbic acid-overproducing transgenic tobacco cells expressing sense RNA for L-galactono-1, 4-lactone dehydrogenase. Planta 220, 854-863. doi: 10.1007/s00425-004-1406-3

Tseng, M. J., Liu, C. W., and Yiu, J. C. (2007). Enhanced tolerance to sulfur dioxide and salt stress of transgenic Chinese cabbage plants expressing both superoxide dismutase and catalase in chloroplasts. Plant Physiol. Biochem. 45, 822-833. doi: 10.1016/j.plaphy.2007.07.011

Ushimaru, T., Nakagawa, T., Fujioka, Y., Daicho, K., Naito, M., Yamauchi, Y., et al. (2006). Transgenic Arabidopsis plants expressing the rice dehydroascorbate reductase gene are resistant to salt stress. J. Plant Physiol. 163, 1179-1184. doi: 10.1016/j.jplph.2005.10.002

Vainonen, J. P., and Kangasjarvi, J. (2014). Plant signaling in acute ozone exposure. Plant Cell Environ. doi: 10.1111/pce.12273. [Epub ahead of print].

Vanderkooi, J. M., Erecinska, M., and Silver, I. A. (1991). Oxygen in mammalian tissue: methods of measurement and affinities of various reactions. Am. J. Physiol. 260, C1131-C1150.

Vanlerberghe, G. C., and McIntosh, L. (1992). Coordinate regulation of cytochrome and alternative pathway respiration in tobacco. Plant Physiol. 100, 1846-1851. doi: $10.1104 /$ pp.100.4.1846

Vendruscolo, E. C. G., Schuster, I., Pileggi, M., Scapim, C. A., Molinari, H. B. C., Marur, C. J., et al. (2007). Stress-induced synthesis of proline confers tolerance to water deficit in transgenic wheat. J. Plant Physiol. 164, 1367-1376. doi: 10.1016/j.jplph.2007.05.001

Wagner, D. E., Przybyla, D., op den Camp, R. G., Kim, C., Landgraf, F., Lee, K. P., et al. (2004). The genetic basis of singlet oxygen-induced stress responses of Arabidopsis thaliana. Science 306, 1183-1185. doi: 10.1126/science.11 03178

Wang, Y. C., Qu, G. Z., Li, H. Y., Wu, Y. J., Wang, C., Liu, G. F., et al. (2010). Enhanced salt tolerance of transgenic poplar plants expressing a manganese superoxide dismutase from Tamarix androssowii. Mol. Biol. Rep. 37, 1119-1124. doi: 10.1007/s11033-009-9884-9

Wang, Y., Wisniewski, M., Meilan, R., Cui, M., and Fuchigami, L. (2006). Transgenic tomato (Lycopersicon esculentum) overexpressing cAPX exhibits enhanced tolerance to UV-B and heat stress. J. Appl. Hortic. 8, 87-90.

Whiteman, S. A., Nuhse, T. S., Ashford, D. A., Sanders, D., and Maathuis, F. J. M. (2008). A proteomic and phosphoproteomic analysis of Oryza sativa plasma membrane and vacuolar membrane. Plant J. 56, 146-156. doi: 10.1111/j.1365313X.2008.03578.x

Willekens, H., Chamnongpol, S., Davey, M., Schraudner, M., Langebartels, C., Van Montagu, M., et al. (1997). Catalase is a sink for H2O2 and is indispensable for stress defense in C3 plants. EMBO J. 16, 4806-4816.

Wrzaczek, M., Brosché, M., and Kangasjarvi, J. (2013). ROS signalling loopsproduction, perception, regulation. Curr. Opin. Plant Biol. 16, 575-582. doi: 10.1016/i.pbi.2013.07.002

Xing, Y., Jia, W., and Zhang, J. (2008). AtMKK1 mediates ABA-induced CAT1 expression and $\mathrm{H}_{2} \mathrm{O}_{2}$ production via AtMPK6-coupled signaling in Arabidopsis. Plant J. 54, 440-451. doi: 10.1111/j.1365-313X.2008.03433.x

Xu, J., Yang, J., Duan, X., Jiang, Y., and Zhang, P. (2014). Increased expression of native cytosolic $\mathrm{Cu} / \mathrm{Zn}$ superoxide dismutase and ascorbate peroxidase improves tolerance to oxidative and chilling stresses in cassava (Manihot esculenta Crantz). BMC Plant Biol. 14:208. doi: 10.1186/s12870-014-0208-4

Xu, W. F., Shi, W. M., Ueda, A., and Takabe, T. (2008). Mechanisms of salt tolerance in transgenic Arabidopsis thaliana carrying a peroxisomal ascorbate peroxidase gene from barley. Pedosphere 18, 486-495. doi: 10.1016/S1002-0160(08) 60039-9 
Yabuta, Y., Maruta, T., and Yoshimura, K. (2004). Two distinct redox signaling pathways for cytosolic APX induction under photo-oxidative stress. Plant Cell Physiol. 45, 1586-1594. doi: 10.1093/pcp/pch181

Yamada, M., Morishita, H., Urano, K., Shiozaki, N., Yamaguchi-Shinozaki, K., Shinozaki, K., et al. (2005). Effects of free proline accumulation in petunias under drought stress. J. Exp. Bot. 56, 1975-1981. doi: 10.1093/jxb/eri195

Yoshimura, K., Miyao, K., Gaber, A., Takeda, T., Kanaboshi, H., Miyasaka, H., et al. (2004). Enhancement of stress tolerance in transgenic tobacco plants overexpressing Chlamydomonas glutathione peroxidase in chloroplasts or cytosol. Plant J. 37, 21-33. doi: 10.1046/j.1365-313X.2003.01930.x

Zelitch, I. (1990). Further studies on $\mathrm{O}_{2}$-resistant photosynthesis andphotorespiration in a tobacco mutant with enhanced catalase activity. Plant Physiol. 92: 352-357. doi: 10.1104/pp.92.2.352

Zhang, Z., Wang, J., Zhang, R., and Huang, R. (2012). The ethylene response factor AtERF98 enhances tolerance to salt through the transcriptional activation of ascorbic acid synthesis in Arabidopsis. Plant J. 71, 273-287. doi: 10.1111/j.1365313X.2012.04996.x
Conflict of Interest Statement: The authors declare that the research was conducted in the absence of any commercial or financial relationships that could be construed as a potential conflict of interest.

Received: 29 November 2014; accepted: 30 December 2014; published online: 15 January 2015.

Citation: Das P, Nutan KK, Singla-Pareek SL and Pareek A (2015) Oxidative environment and redox homeostasis in plants: dissecting out significant contribution of major cellular organelles. Front. Environ. Sci. 2:70. doi: 10.3389/fenvs.2014.00070

This article was submitted to Environmental Toxicology, a section of the journal Frontiers in Environmental Science.

Copyright (c) 2015 Das, Nutan, Singla-Pareek and Pareek. This is an open-access article distributed under the terms of the Creative Commons Attribution License (CC BY). The use, distribution or reproduction in other forums is permitted, provided the original author(s) or licensor are credited and that the original publication in this journal is cited, in accordance with accepted academic practice. No use, distribution or reproduction is permitted which does not comply with these terms. 